\title{
Diagnosis and treatment of carotid artery stenosis
}

\begin{abstract}
In developed countries, stroke is the primary cause of invalidity and the third most common cause of mortality. The cause of stroke in about $30 \%$ of cases are emboli emerging from the heart, $25 \%$ of cases are caused by atherosclerosis of large cerebral arteries $(85 \%$ are due to carotid atherosclerotic disease), about $20 \%$ of cases by occlusions of small cerebral arteries (lacunar infarct), $20 \%$ of cases by multiple or unspecified causes, and about $5 \%$ of cases by vasculopathies, hypercoagulable conditions and hematological diseases. Stroke is, therefore, a very significant cause of mortality, and stenotic-occlusive disease of carotid arteries is the cause of stroke in about $20-25 \%$ of patients. Numerous diagnostic methods were developed for efficient verification of carotid pathology due to its great importance in the etiology of stroke. Doppler ultrasound examination of carotid arteries is a dominant non-invasive imaging method in the diagnosis of carotid pathology and is often indicated in practice. Ultrasound is an excellent method for early discovery, quantification and classification of atherosclerotic changes. Today it is a method of choice for establishing the success rate of the surgical procedures involving the carotid arteries. However, there are also other diagnostic procedures available today, such as CT-angiography, MR-angiography and digital subtraction angiography. Pharmacological treatment and surgical procedures like carotid endarterectomy (CEA) and endovascular treatment (CAS-carotid artery stenting, CBA-carotid balloon angioplasty) have substantially contributed to the success rate of treating stenotic-obliterative changes in carotid arteries.
\end{abstract}

Keywords: stenotic-occlusive disease of carotid arteries, diagnosis, treatment
Volume 7 Issue $3-2017$

\author{
Borislav Radic \\ Department of Neurology, University Hospital Center Zagreb, \\ Croatia
}

Correspondence: Borislav Radic, Department of Neurology, University Hospital Center Zagreb, Kišpatićeva 12, I Zagreb, Croatia, Tel +385 I/2376-4l4; +3859 I/798-6045,

Email boris.radic105@gmail.com

Received: April 23, 2017 | Published: August 10, 2017

\section{Introduction}

Stroke is a very important cause of disability and mortality, and atherosclerotic stenotic-occlusive carotid artery disease accounts for about $20-25 \%$ of the cases. Doppler ultrasound examination of carotid arteries is the dominant non-invasive imaging method for assessing the degree of stenosis of the internal carotid arteries as well as in selecting candidates for surgical or endovascular procedures. ${ }^{1,2}$ In addition to its use in the triage of patients slated for medical, surgical or endovascular treatment, doppler ultrasound examination of carotid arteries is routinely performed in all patients hospitalized for cardiosurgical procedures. Ultrasound is an excellent method for early detection, quantification and characterization of atherosclerotic changes in the extracranial arteries of the neck, especially in the carotid bifurcation and the neck segment of internal carotid artery (ACI). Doppler ultrasound also plays a very important role in postoperative monitoring of the success rate following surgical procedure and diagnosis of postoperative restenotic changes. Other diagnostic methods such as CT angiography and MR angiography are used after a positive doppler ultrasound examination of carotid arteries. Digital Subtraction Angiography (DSA) is thought to be a gold standard in diagnosis and establishing degree of ACI stenosis and other changes. It is an invasive method that requires intraarterial application or intravenous contrast, puncture and catheterization of major arteries, exposes the patient to ionizing radiation and has a risk of neurological and non-neurological complications. ${ }^{3}$

\section{Clinical signs and causes}

Clinical symptoms of carotid artery disease may manifest themselves as stroke or transient ischemic attack. The most cases of carotid artery stenosis are caused by atherosclerotic plaque as part of generalized atherosclerotic disease. The most important noneaterosclerotic cause of carotid artery stenosis is carotid artery dissection with fibromuscular dysplasia and arteritis being less prevalent. Other causes include neck radiotherapy, connective tissue disease and trauma. Carotid artery dissection may occur due to selective catheterization of carotid arteries or may be come about as part of inborn artery wall defects, such as Marfan or Ehlers-Danlos disease. The stenotic changes caused by fibromuscular dysplasia should primarily considered in younger patients with atherosclerosis. In the case of multiple stenosis or extracranial cervical arteries occlusion, especially in younger women (with elevated erythrocyte sedimentation in the laboratory findings) Takayasu arteritis should be considered as primary diagnosis. ${ }^{4,5}$

\section{Diagnosis}

A clear division between symptomatic and asymptomatic carotid stenosis is of fundamental importance when considering the commencement the method of treatment. Doppler ultrasound examination has an important role in the diagnosis of cerebrovascular diseases since allows noninvasive and objective diagnostic evaluation as well as assessment of brain hemodynamics. Color Doppler ultrasound provides extremely important information about the condition of carotid and vertebral arteries, the thicknesses of the intimamedia complex as a fundamental marker of elevated cardiovascular risk and clearly shows accommodation, length, structure and content of atherosclerotic plaques. ${ }^{6,7}$ If the stenotic process involving the extra or intracranial blood vessels is ultrasonically determined, additional neuroradiological diagnostic procedures should be performed such as CTA or MRA and in some cases DSA. The sensitivity of MRA is 91$95 \%$ and the specificity is $88-99 \%$. The advantage of this procedure is that it enables an insight into the entire artery structure and is less dependent on the diagnostic experience of the evaluator. Additionally gadolinic contrast has a significantly lower incidence of nephropathy and allergic reactions than iodine contrast used in DSA. The deficiencies include smaller sensitivity in moderate stenosis, inability to differentiate between subocllusion and occlusion, relatively high price and limited availability as well as fact that in some cases it is difficult to perform, such as patients with severe general condition, patients with claustrophobia or patients with a heart electrostimulator 
or embedded defibrillator. CTA on the other hand provides limited insight into the arterial wall or the surrounding structures. The sensitivity of the CTA procedure is $77 \%$ and specificity is $95 \%$. It is particularly useful in cases where ultrasound provides limited insight (e.g. tortuosity and arterial calcification). It also enables visualization of the stenosis level ranking from subocclusion to occlusion. The disadvantages of the procedure include the use of x-rays and the need for contrast which may lead to contrast-induced nephropathy. Additionally, the precision of the stenosis assessment may be limited by the presence of calcifications in the blood vessel walls. ${ }^{8}$

\section{Surgical treatment}

Prompt evaluation and triage of patients with symptomatic carotid artery stenosis is essential to minimize the risk of early recurrent cerebrovascular events. Prospective studies have shown that the risk of ipsilateral stroke is highest within the first 90 days, and especially within the first month following a TIA. ${ }^{9}$ Urgent commencement of treatment can reduce this risk by up to $80 \%{ }^{9}$

A scoring system for evaluation of the short-term risk of ipsilateral stroke after a TIA $^{10}$ (Table 1) is named after the acronym $A B C D 2$ : age, blood pressure, clinical features, duration of symptoms, and diabetes mellitus. A patient with a TIA and an ABCD2 score of 0 to 3 has a $1.2 \%$ risk of stroke within 7 days, as opposed to a $5.9 \%$ risk with a score of 4 or 5 and an $11.7 \%$ risk with a score of 5 or $6 .{ }^{10}$ High $\mathrm{ABCD} 2$ scores also seem to correlate with moderate or severe carotid artery stenosis. ${ }^{9}$

$\mathrm{ABCD}^{2}$ is an acronym for the 5 factors of the scoring system. ${ }^{10}$

Table I Criteria and points of the $A B C D^{2}$ Scoring system

\begin{tabular}{ll}
\hline Factor & Points \\
\hline Age $\leq 60 \mathrm{y}$ & $\mathrm{I}$ \\
Blood Pressure $\geq 140 / 90 \mathrm{~mm}$ of $\mathrm{Hg}$ & \\
Clinical Features & \\
Unilateral weakness & 2 \\
Speech impairment without weakness & $\mathrm{I}$ \\
Duration of transient ischemic attack & \\
$\geq 60 \mathrm{~min}$ & 2 \\
I0-59 min & $\mathrm{I}$ \\
Diabetes Mellitus & $\mathrm{I}$
\end{tabular}

Indications for the treatment of patients with carotid artery stenosis are based on several aspects of neurological symptomatology and the degree of carotid stenosis, comorbidity as well as high risk for vascular and local anatomical stenosis. Carotid endarterectomy (CEA), a surgical therapeutical procedure is associated with low mortality and low percentage of perioperative complications. The results of NASCET studies highlight the key role of CEA in the ICA stenosis measuring over 70\%. CEA is absolutely indicated in patients with stenosis measuring $70 \%-99 \%$ and should only be performed in centers with established perioperative risk of less than $<6 \% .{ }^{9} \mathrm{CEA}$ should be performed as soon as possible following the ischemic event, ideally within two weeks. In patients with symptomatic stenosis of $70 \%$ to $99 \%$, the absolute risk reduction decreased from $23 \%$ to $16 \%$ and then to $8 \%$ when patients were treated within 2 weeks, between 2 and 4 weeks, and after 4 weeks, respectively. The inverse benefit of CEA with increasing time from the qualifying ischemic event was particularly evident in women. ${ }^{11}$ In fact, the benefit of treatment was lost in women when treatment was delayed more than 2 weeks. ${ }^{11}$

In patients with stenosis 50-69\% CEA should be performed only in centers with perioperative complication risk $<6 \%$. Complex analyzes of randomized controlled trials support the fact that the effect of
CEA is maximized in patients who are operated within 2 weeks from the neurological incident, such as brain stroke or TIA. ${ }^{11}$ Due to the potential benefits of CEA in primary stroke prevention, the screening of asymptomatic subjects has led to the increase in the detection rates of carotid stenosis in the general population. Therefore, it is assumed that CEA's availability will increase over time and in the next decade CEA will become a safe approach that will be applied within 24 hours of a neurological event. Medication therapy is recommended in the cases of stenoses measuring less than $50 \%$ even if they are symptomatic.

Traditionally, surgeons have been reluctant to operate within 1 month of a qualifying TIA or a minor stroke because of the perceived higher risk of periprocedural complications ${ }^{12}$. However, subgroup analysis of the RCTs (randomized controlled trial) showed that the perioperative risks of stroke and death were not increased in patients who underwent an operation within 2 weeks of the qualifying event ${ }^{11}$. The guidelines for secondary stroke prevention recommend that CEA should be performed within 2 weeks for patients presenting with a TIA or minor stroke. ${ }^{13,14}$

The rate of stroke occurrence or death among symptomatic patients following carotid endarterectomy in the CREST trial (3.2\%) was lower than the corresponding percentage in SPACE study (6.3\%) and was similar to the corresponding percentage in EVA-3S (3.9\%) as well as that in ICSS $(3.4 \%){ }^{15}$

\section{Endovascular treatment}

Carotid artery stenting (CAS) has been initially used as an alternative treatment option in patients not eligible for surgery. Numerous non-randomized and some randomized studies have assessed the safety and efficacy of CAS in so-called high risk patients. ${ }^{16-20}$ Although CAS has been recommended in specialized subsets of patients ${ }^{21-23}$ such as restenosis after CEA, radiation-induced carotid stenosis, increased cardiopulmonary risk or unfavorable neck anatomy as well as in higher risk patients, the appropriateness of its use in patients with conventional risk remains an unsolved matter. The potential benefits of endovascular treatment (angioplasty with or without stent implantation) as an alternative to carotid endarterectomy were first highlighted by the Carotid and Vertebral Artery Transluminal Angioplasty Study (CAVATAS). ${ }^{24}$ The Stenting and Angioplasty with Protectionin Patients at High Risk for Endarterectomy (SAPPHIRE) trial $^{16}$ is the only randomized trial that specifically enrolled high risk patients to compare CEA and CAS with embolic protection devices. The primary endpoint (the composite of MI, stroke, or death within 30 days plus death because of neurological causes or ipsilateral stroke between 31 days and 1 year) occurred in $12.2 \%$ of patients assigned to CAS and $20.1 \%$ of those assigned to CEA. The investigators of the SAPPHIRE trial concluded that CAS was not inferior to CEA. The Stent-Protected Angioplasty versus Carotid Endarterectomy (SPACE) trial randomized 1200 symptomatic patients. The incidence of ipsilateral stroke or death at 30 days was the primary endpoint of the study and did not differ between the groups $(6.3 \%$ for CEA vs. $6.8 \%$ for CAS) ${ }^{25}$ The SPACE trial failed to prove the non-inferiority of CAS due to the insufficient sample size. However, no differences were found between CAS and CEA in respect to the prevention of recurrent cerebrovascular events after treatment of severe symptomatic carotid artery stenosis at 2 years. The large trials like CREST have shown that with adequate training, physicians can perform both CAS and CEA with low complication rates. ${ }^{26}$

Taken together, the results of the CAVATAS, SAPPHIRE, SPACE, EVA-3S and CREST studies suggest that CAS is as effective as CEA for the medium-term prevention of ipsilateral stroke, at least 
for the first 4 years after the procedure, albeit with limited statistical power. The rate of stroke or death among symptomatic patients after carotid artery stenting in the CREST trial $(6.0 \%)$ was lower than the corresponding rates in the SPACE $(6.8 \%$, not including nonipsilateral stroke), EVA-3S and ICSS (7.4\%) trials. ${ }^{26}$

Endovascular treatment such as carotid balloon angioplasty with or without stents, have primarily in the treatment of stroke. They are indicated in noninvasively proven stenosis greater than $70 \%$. CAS is a less invasive method than CEA. The procedure is performed under local anesthesia, without throat dissection and possible risk of peripheral nerve damage and is less painful. Absolute indications for CAS include patients with symptomatic ACI stenosis, postoperative CEA restenosis, post-radiation stenosis, anatomically elevated ACI stenosis (bifurcation), tandem lesions (associated ACI and ACC) and fibromuscular dysplasia. Relative indications for CAS are rapidly progressing asymptomatic stenosis of carotid arteries and high grade double stenosis carotid arteries. ${ }^{16}$ Some studies show the advantage of CAS in patients with advanced coronary and renal disease, chronic obstructive disease and contralateral ACI occlusion. Increased risk for periprocedural complications after endovascular treatment was observed in patients older than 80 years with advanced chronic renal insufficiency, complex atherosclerotic disease of carotid bifurcation, multiple lesions or angulations of ACI, pronounced tortuosities or calcifications of aortic artery or carotid bifurcation, long subtotal stenosis of the ACI and in patients who have had severe stroke in the previous 4-6 weeks or have a poor femoral approach. Absolute contraindications for endovascular treatment include patients with intolerance to acetylsalicylic acid or clopidogrel, circulatory calcification of ACI, intraluminal thrombus, chronic ACI occlusion, and presence of intracranial aneurysm or AV malformation.

\section{Medical treatment}

A medical approach to the treatment of carotid artery stenosis implies a combination of non-invasive procedures consisting of education, changes in habits, healthy eating, dietary measures and drug administration. Drug therapy is based on the smoking abstinence and treatment of arterial hypertension, hypercholesterolemia, diabetes, as well as the use of antithrombotic drugs such as aspirin and clopidogrel. For carotid artery stenosis-measuring less than $<50 \%$ only drug treatment is indicated except when in the case of highly unstable atherosclerotic plaque with high risk for causation of thromboembolic incidents. ${ }^{27}$ In some cases of asymptomatic stenosis measuring up to $70 \%$ and developed collateral vasculature can be used in the treatment coupled with regular doppler ultrasound control examination.

\section{Carotid endarterectomy or stenting?}

The standard procedure for invasive treatment of carotid artery stenosis is CEA. Compared with CEA, CAS has the advantage that it can be done in patients under mild sedation, it requires no large surgical incisions, carries no risk of cranial nerve palsy and has fewer cardiovascular complications. Over the years, CEA has been shown to be safe and effective in preventing ipsilateral strokes with longlasting effect. For its acceptance as a valid alternative to CEA, CAS must fulfill the same criteria of safety and effectiveness. The safety of CAS compared to CEA has been studied in several RCTs. ${ }^{28}$ These recent studies have several common features, including prospective data collection, outcome assessment by independent neurologists, and similar end points of myocardial infarction, stroke, and death within 30 days of the procedure. Although the results of these studies have not unequivocally established the safety superiority of CAS vs CEA, they have identified high-risk subgroups. It was thought that the CAS procedure, being less invasive than CEA, could be advantageous in older patients. However, paradoxically, subgroup analyses of prospective studies have consistently shown that greater age is associated with a higher risk of periprocedural morbidity and death after CAS but not necessarily following CEA. ${ }^{29-31}$ Additionally neurological symptoms occuring within 2 weeks of the procedure are associated with increased complication rates. ${ }^{29}$

A meta-analysis of published RCTs indicated that the risk of stroke within 30 days of the intervention was slightly higher in the CAS than the CEA group. ${ }^{32}$ However, the long-term (2-3 years) effectiveness of CAS has been demonstrated by randomized studies showing that the long-term incidence of ipsilateral strokes is not different between CAS and CEA. ${ }^{23,33,34}$ Data on the incidence of restenosis are preliminary, but restenosis rates reported after CAS are relatively low. ${ }^{23,33}$ More importantly, most restenoses are asymptomatic and therefore of unclear clinical importance. Restenosis rates may also decrease as the stent technology continues to improve. While clinicians await the conclusion of the large RCTs, CAS should be considered in patients with indication for carotid revascularization and high surgical risk.

\section{Conclusion}

Atherosclerotic stenotic-occlusive carotid artery disease is one of the most common causes of stroke (20-25\%). Due to this fact, timely diagnosis of stenotic-occlusive changes in carotid arteries plays a very important role in early detection. This approach includes the use of modern diagnostic methods, medical treatment and surgical or endovascular treatment. Numerous diagnostic methods have been developed for early detection of carotid pathology: ultrasound, DSA, CTA, MRA. More recently, doppler ultrasound examination has assumed one of the most important roles in the diagnosis of cerebrovascular disease allowing for noninvasive and objective diagnostics and evaluation of brain hemodynamics, monitoring of the operative treatment success and detecting postoperative complications. Selection of treatment options in carotid artery disease should be done by an interdisciplinary team consisting of a neurologist, vascular surgeon, anesthesiologist and intervention radiologist.

The recent results of large randomized clinical trials indicate improving outcomes for patients requiring treatment for carotid artery stenosis, either through interventional or medical treatment. While medical therapy alone is considered the gold standard for patients with asymptomatic stenosis of carotid artery, intervention confers an outcome benefit in symptomatic patients. In the last few years CAS has emerged as a valid alternative to CEA, which is still considered as the best treatment option. The results of randomized trials have not shown consistent outcome differences between CAS and CEA. CAS is associated with heightened periprocedural risks of stroke and death, while CEA is associated with increased incidence of myocardial infarction and cranial nerve paralysis. CAS may be superior to CEA in certain groups of patients, such as those exposed to previous neck surgery or radiation injury. When performed in conjunction with an embolic protection device, the risks associated with CAS may be lower than those associated with CEA in patients at elevated risk of surgical complications. The selection of patients for either CEA or CAS may require attention to age, with patients younger than 60 years having a slightly better outcome with CAS, patients older than 70 years having a better outcome with CEA and those younger than 70 years having an equivalent or better aggregate outcome with CAS. Follow-up of ongoing clinical trials will provide new data regarding relative costs and benefits of CAS versus CEA, long-term restenosis rates and a better definition of subgroups that may benefit from specific interventions. The indications for CEA and CAS have not been firmly established. CEA is more appropriate than 
CAS in the case of soft atherosclerotic plaques present in the stenotic lesion, since such soft plaques are associated with a high incidence of ischemic complications during CAS. ${ }^{35}$ However, the rapid evolution in CAS technique and materials suggests a great potential for CAS to improve outcomes and demonstrate superiority compared to CEA in the foreseeable future.

\section{Acknowledgments}

None.

\section{Conflicts of interest}

None.

\section{Funding}

None.

\section{References}

1. Feigin VL, Mensah GA, Norrving B, et al. Atlas of the Global Burden of Stroke (1990-2013): The GBD 2013 Study. Neuroepidemiology. 2015;45(3):230-236.

2. Morgan JA, Ackerman RH, Romero JM. Noninvasive imaging evaluation of carotid artery occlusive disase. Applied radiology. 2014;33:8-16.

3. Nederkoorn PJ, van der Graaf Y, Hunink MG. Duplex Ultrasound and Magnetic Resonace Angiography Compared With Digital Subtraction Angiography in Carotid Artery Stenosis: a systematic review. Stroke. 2003;34(5):1324-1332.

4. Sutton D. Textbook of Radiology and imaging Seventh edition Churcill Livingstone Elsevier science. 2003.

5. Garg SK, Mohan S, Kumar S. Diagnostic value of 3D contrastenhanced magnetic resonance angiography in Takayasu arteritis-a comparative study with digital subtraction angiography. Eur Radiol. 2011;21(8):1658-1666.

6. Grant EG, Benson CB, Moneta GL, et al. Carotid artery stenosis: grayscale and Doppler US diagnosis-Society of Radiologists in Ultrasound Consensus Conference. Ultrasound. 2003;19(4):190-198.

7. De Bray JM, Baud JM, Dauzat M. Consensus concerning the morphology and the risk of carotid plaques. Cerebrovasc Dis. 1997;7:289-296.

8. Higwala D, Kesavadas Ch, Sylaja PN, et al. Multimodality imaging of carotid atherosclerode plaque: Going beyond stenosis. Indian J Radiol Imaging. 2013;23(1):26-34.

9. Rothwell PM, Giles MF, Chandratheva A, et al. Effect of urgent treatment of transient ischaemic attack and minor stroke on early recurrent stroke (EXPRESS study): a prospective population-based sequential comparison. Lancet. 2007;370(9596):1432-1442.

10. Johnston SC, Rothwell PM, Nguyen Huynh MN, et al. Validation and refinement of scores to predict very early stroke risk after transient ischaemic attack. Lancet. 2007;369(9558):283-292.

11. Rothwell PM, Eliasziw M, Gutnikov SA, et al. Sex difference in the effect of time from symptoms to surgery on benefit from carotid endarterectomy for transient ischemic attack and nondisabling stroke. Stroke. 2004;35(12):2855-2861.

12. Naylor AR. Delay may reduce procedural risk, but at what price to the patient. Eur J Vasc Endovasc Surg. 2008;35(4):383-391.

13. Sacco RL, Adams R, Albers G, et al. Guidelines for prevention of stroke in patients with ischemic stroke or transient ischemic attack: a statement for healthcare professionals from the American Heart Association/ American Stroke Association Council on Stroke: co-sponsored by the Council on Cardiovascular Radiology and Intervention: the American of Neurology affirms the value of this guideline. Stroke. 2006;37(2):577-617.
14. Barnett HJ, Taylor DW, Eliasziw M, et al. Benefit of carotid endarterectomy in patients with symptomatic moderate or severe stenosis. North american symptomatic carotid endarterectomy trial collaborators. N Engl J Med. 1998;339(20):1415-1425.

15. Brott TGM, Hobson RW, Howard G, et al. Stenting versus Endarterectomy for Treatment of Carotid Artery Stenosis. $N$ Engl J Med. 2010;363(1):11-23.

16. Yadav JS, Wholey MH, Kuntz RE, et al. Protected carotid-artery stenting versus endarterectomy in high-riskpatients. $N$ Engl $\mathrm{J}$ Med. 2004;351(15):1493-1501.

17. Al-Mubarak N, Roubin GS, Gomez CR, et al. Carotidartery stenting in patients with high neurologic risks. AmJ Cardiol. 1999;83(9):1411-1413.

18. Malek AM, Higashida RT, Phatouros CC, et al. Stent an-gioplasty for cervical carotid artery stenosis in high-risksymptomatic NASCETineligible patients. Stroke. 2000;31(12):3029-3033.

19. Waigand J, Gross CM, Uhlich F, et al. Elective stentingof carotid artery stenosis in patients with severe coronaryartery disease. Eur Heart $J$. 1998;19(9):1365-1370.

20. Teitelbaum GP, Lefkowitz MA, Giannotta SL. Carotidangioplasty and stenting in high-risk patients. Surg Neu-rol. 1998;50(4):300-311.

21. Hobson RW, Mackey WC, Ascher E, et al. Manage-ment of atherosclerotic carotid artery disease: clinicalpractice guidelines of the Society for Vascular Surgery. J Vasc Surg. 2008;48(2):480-486.

22. Gurm HS, Yadav JS, Fayad P, et al. Long term results ofcarotid stenting versus endarterectomy in high risk patients. $N$ Engl $J$ Med. 2008;358(15):1572-1579.

23. Eckstein HH, Ringleb P, Allenberg JR, et al. Results of the Stent Protected Angioplasty versus Carotid En-darterectomy (SPACE) study to treat symptomaticstenoses at 2 years: a multinational prospective randomised trial. Lancet Neurol. 2008;7(10):893-902.

24. Endovascular versus surgical treatment in patients withcarotid stenosis in the Carotid and Vertebral ArteryTransluminal Angioplasty Study (CAVATAS): a ran-domised trial. Lancet. 2001; 357(9270):1729-1737.

25. Group SC, Ringleb PA, Allenberg J, et al. 30 day resultsfrom the SPACE trial of stent-protected angioplasty ver-sus carotid endarterectomy in symptomatic patients: arandomised non-inferiority trial. Lancet. 2006;368(9543):1239-1247.

26. Brott TG, Halperin JL, Abbara S, et al. Guideline on the Management of Patients With Extracranial Carotid and Vertebral Artery Disease. Stroke. 2011;3(2):100-130.

27. Lanzino G, Robinstein AA, Brown RD. Treatment of Carotid Artery Stenosis: Medical, Surgery or Stenting. Mayo Clinic Proc. 2009;84(4):362-387.

28. Goodney PP, Powell RJ. Carotid artery stenting: what have we learned from the clinical trials and registries and where do we go from here. Ann Vasc Surg. 2008;22(1):148-158.

29. Gray WA, Yadav JS, Verta P, et al. The CAPTURE registry: predictors of outcomes in carotid artery stenting with embolic protection for high surgical risk patients in the early post-approval setting. Catheter Cardiovasc Interv. 2007;70(7):1025-1033.

30. Hobson RW, Howard VJ, Roubin GS, et al. CREST Investigators Carotid artery stenting is associated with increased complications in octogenarians: 30-day stroke and death rates in the CREST lead-in phase. J Vasc Surg. 2004;40(6):1106-1111.

31. Stingele R, Berger J, Alfke K, et al. SPACE investigators Clinical and angiographic risk factors for stroke and death within 30 days after carotid endarterectomy and stent-protected angioplasty: a subanalysis of the SPACE study. Lancet Neurol. 2008;7(3):216-222. 
32. Murad MH, Flynn DN, Elamin MB, et al. Endarterectomy vs stenting for carotid artery stenosis: a systematic review and meta-analysis. $J$ Vasc Surg. 2008;48(2):487-493.

33. Gurm HS, Yadav JS, Fayad P, et al. Long-term results of carotid stenting versus endarterectomy in high-risk patients. $N$ Engl J Med. 2008;358(15):1572-1579.
34. Mas JL, Trinquart L, Leys D, et al. Endarterectomy Versus Angioplasty in Patients with Symptomatic Severe Carotid Stenosis (EVA-3S) trial: results up to 4 years from a randomised, multicentre trial. Lancet Neurol. 2008;7(10):885-892.

35. Tsukahara T. Surgical Treatment of Carotid Artery Stenosis. Acta Neurochir Suppl. 2006;123:109-114. 